\title{
Evaluation of atherogenic laboratory markers in Behçet's disease patients with vascular involvement
}

\author{
Çiğdem Yücel ${ }^{1}$, Ahmet Omma ${ }^{2}$, Erdim Sertoğlu ${ }^{3}$, Sevilay Sezer ${ }^{1}$, Turan Turhan ${ }^{1}$, Taner Özgürtaş ${ }^{3}$
}

\author{
${ }^{1}$ Department of Clinical Biochemistry, Ankara Numune Training and Research \\ Hospital, Altındağ, Ankara, Turkey \\ 2Department of Rheumatology Ankara Numune Training and Research Hospital, \\ Altındağ, Ankara, Turkey \\ ${ }^{3}$ Department of Clinical Biochemistry, Ankara Gülhane Training and Research \\ Hospital, Etlik, Ankara, Turkey
}

Submitted: 22 June 2018

Accepted: 21 August 2018

Arch Med Sci 2020; 16 (3): 531-537

DOI: https://doi.org/10.5114/aoms.2018.79139

Copyright @ 2019 Termedia \& Banach

\begin{abstract}
Introduction: Behçet's disease is a chronic inflammatory vasculitis presenting with immunological and endothelial changes. The aim of the present study is to evaluate blood levels of diagnostic markers which can be used in Behçet's patients with vascular involvement.

Material and methods: Fifty Behçet's patients (22 with vascular involvement) and 30 healthy controls were included in the study. High-sensitivity C-reactive protein (hSCRP), erythrocyte sedimentation rate (ESR), tumor necrosis factor- $\alpha$ (TNF- $\alpha$ ), apolipoprotein A1 (apoA1), apolipoprotein B (apoB), high-density lipoprotein (HDL), low-density lipoprotein ( $L D L)$, triglyceride, total cholesterol, Lp-plA-2, homocysteine and ischemia modified albumin (IMA) levels were analyzed. Statistical analysis was performed with the SPSS program version 11.0. $p<0.05$ was accepted as statistically significant.

Results: hsCRP, TNF- $\alpha$, homocysteine, IMA, apoA1, apoB, HDL, Lp-pla2 and ESR levels in patient and control groups were significantly different $(p<0.001, p=0.001, p<0.001, p<0.001, p=0.005, p<0.001, p<0.001$, $p<0.001$ and $p=0.003$ respectively). In Behçet's patients with vascular involvement, homocysteine, TNF- $\alpha$ and Lp-pla2 levels were significantly higher than in Behçet's patients without vascular involvement ( $p=0.035, p=0.010$ and $p<0.001$ respectively).

Conclusions: Increased levels of inflammatory and atherogenic markers in Behçet's patients are an expected outcome due to the inflammatory nature of the disease. Especially, elevated levels of homocysteine, TNF- $\alpha$ and Lppla2 make them candidate diagnostic tools to be helpful in clinical evaluation of Behçet's disease patients with vascular involvement.
\end{abstract}

Key words: Behçet's disease, inflammation, vascular involvement, atherosclerosis.

\section{Introduction}

Behçet's disease (BD) is a chronic, multisystemic disease characterized by recurrent acute inflammatory attacks. The disease is accompanied by mucocutaneous, ocular, articular, vascular and neurological involvement along with involvement of all other organ systems. Although the exact etiology of the disease is still unknown, three major pathophysiological changes involved in BD are neutrophil hyperfunction, vasculitis and the

\author{
Corresponding author: \\ Çiğdem Yücel \\ Ankara Numune Training \\ and Research \\ Hospital Biochemistry Clinic \\ Talatpasa Blv. 4, Altındağ \\ Ankara, Turkey \\ Phone: +905335101570 \\ Fax: +90 3125083245 \\ E-mail: yucelcigdem80@ \\ gmail.com
}


autoimmune response [1-3]. Clinical examination is still the major component in diagnosis as a consensus has not been reached in terms of common histological, radiological and laboratory findings presented in BD patients [4]. Endothelial damage has a key role in the pathogenesis and immunopathology of $\mathrm{BD}$ as it causes the extravasation of leukocytes and other blood components. Focal lipid deposition is known to take place in the arterial intima layer as a result of endothelial dysfunction. The inflammatory process also includes major immune system cells.

Tumor necrosis factor- $\alpha$ (TNF- $\alpha$ ) is a lymphokine primarily released from activated monocytes and macrophages. This pro-inflammatory cytokine has also been shown to take place in BD pathogenesis by affecting lipid metabolism as it causes endothelial nitric oxide regeneration and increased circulating levels of non-esterified fatty acids. Oxidative stress and atherogenic dyslipidemia are known to have a synergistic effect on atherosclerosis and cardiovascular disease progression. The underlying pathogenesis of most clinical findings in $B D$ has proven to be vasculitis [5-8].

The enzyme lipoprotein-associated phospholipase A2 (Lp-pla2) is a member of intracellular and secretory phospholipases as it hydrolyses the phospholipid ester bond of cellular membranes and lipoproteins. Lp-pla2 is formed in atherosclerotic plaques by macrophages and foamy cells, and its major association in blood is with LDL cholesterol. Being a specific marker of inflammation in the vascular cluster and having low biological variation, Lp-pla2 can be considered as a candidate marker for cardiovascular and vascular involvement in BD patients [9].

As atherosclerosis is considered as an inflammation-mediated process causing focal lipid accumulation in vessel walls by complex interactions between leukocytes and platelets, the role of serum lipids and lipoproteins is significant in this inflammatory process. Apolipoproteins are one of the major components of lipoproteins, apolipoprotein $B(a p o B)$ is found atherogenic lipoproteins like low-density lipoprotein (LDL) and very low-density lipoprotein (VLDL), while apolipoprotein $A 1$ (apoA1) is found in the structure of the anti-atherogenic cholesterol high-density lipoprotein (HDL). That is why an increase in apoB/apoA1 ratio is considered to be related to progression of cardiovascular disease and a tendency for atherosclerosis $[10,11]$.

C-reactive protein (CRP) and erythrocyte sedimentation rate (ESR) are non-specific systemic inflammation markers. High-sensitivity CRP (hsCRP) is known to be synthesized in the vascular endothelium and also atherosclerotic plaques and higher hsCRP plasma concentration is thought be a cause of atherosclerosis. As its levels are also elevated in subclinical inflammation, hsCRP testing is recommended for both determining individuals with cardiovascular disease (CVD) development risk and CVD prevention (treatment complications and evaluation of treatment efficacy). hsCRP is routinely used for individual cardiovascular risk assessment and its combined usage with lipid profile, lipoproteins and other cardiovascular risk markers such as Lp-pla2 gives a better evaluation of cardiovascular risk $[12,13]$.

Ischemia modified albumin (IMA) is "N-terminal modified albumin" in which albumin's capacity to bind heavy metals such as cobalt and nickel is reduced. Although IMA was initially defined as a marker for myocardial ischemia, recent studies have shown that IMA can be related to oxidative stress with or without coronary ischemia $[14,15]$.

Homocysteine is a sulfur-containing derivative of methionine and is produced in all cells through the normal methylation process of this essential amino acid. Homocysteine is known to have various effects on vascular cells such as reducing nitric oxide release from endothelial cells, producing superoxide and hydrogen peroxide, increasing reactive oxygen species and enhancing thromboxane A2 formation in platelets. Increased levels of the endogenously synthesized amino acid homocysteine is also known to take place in formation of oxidized LDL, which has important role in atherosclerosis. Additionally, homocysteine increases blood viscosity, which in turn causes risk of thrombus formation [16, 17].

Vasculitis is the main pathological finding in BD. This disease is unique among vasculitides as it has a significant thrombotic tendency associated with vascular inflammation. Almost $40 \%$ of BD patients, the majority of them being young males, are known to have vascular complications. Vascular involvement is a major cause of mortality and morbidity and is diagnosed by imaging evaluated together with clinical signs $[18,19]$. Despite the detailed knowledge on different mechanisms of vascular inflammation at the molecular level, no specific laboratory markers have yet been demonstrated to be related to vascular involvement in BD.

Various inflammatory laboratory markers have proven to be helpful in follow-up of BD patients. But as the disease basically progresses as a vasculitis, the atherogenic processes occurring in the vascular endothelium gains much importance in disease progression. As vascular involvement is an important cause of morbidity in BD patients, it is of critical importance to find candidate laboratory markers for vascular BD patients. The present study aims to evaluate blood levels of diagnostic laboratory markers which can be used in routine follow-up of Behçet's patients, but the major goal of the study is 
to discover inflammatory and/or atherogenic laboratory markers to discriminate between $\mathrm{BD}$ patients with and without vascular involvement.

\section{Material and methods}

\section{Study population}

Fifty patients with BD according to the International Study Group criteria [20] and 30 age- and sex-matched healthy controls were included. Patients and controls were excluded if they had one of the following combined diseases/situations: 1) concomitant autoimmune or autoinflammatory disease; 2) acute or chronic infection; 3) malignancy; 4) systemic diseases such as diabetes mellitus and heart failure; 5) pregnancy or postpartum 6 months. Twenty-two out of 50 Behçet's patients had vascular involvement. Twenty-seven out of 50 patients were active and 23 were inactive.

Demographic features and clinical characteristics were recorded. Disease activity was assessed with the Turkish version of the BD Current Activity Form (BDCAF) [21]. The items of this activity include headache, oral ulcers, genital ulcers, erythema, skin pustules, arthralgia, arthritis, intestinal involvement, new eye involvement, new nervous system involvement, and vascular involvement (venous thrombosis and/or arterial aneurysms). The BDCAF score was calculated by adding up the scores on each item and ranged from 0 to 12 . Medical approval for the study protocol was given by the Ethics Committee of Ankara Numune Training and Research Hospital with the approval number $1461 / 2017$. The research protocol complies with the 2000 Declaration of Helsinki and written informed consent was obtained from all participants.

\section{Laboratory analysis}

Venous blood samples were collected from the participants after $12 \mathrm{~h}$ of fasting and were centrifuged at $1500 \mathrm{~g}$ for $10 \mathrm{~min}$. Separated sera were aliquoted into Eppendorf tubes and stored at $-80^{\circ} \mathrm{C}$ until the time of analysis.

Serum levels of TNF- $\alpha$ and Lp-pla2 were detected with a commercial ELISA (double antibody sandwich ELISA method) test kit (DiaSource ImmunoAssays S.A., Belgium and BlueGene Comp, Hong Kong respectively) according to the manufacturers' protocol. HDL, cholesterol, triglyceride and homocysteine measurements were made with the enzymatic colorimetric method, and LDL was calculated according to the Friedewald formula.

Apolipoprotein B, apoA1 and hsCRP values were detected with the immunoturbidimetric method. ESR was studied with the Westergren method.

Ischemia modified albumin was measured with Bar-Or et al.'s colorimetric method [22] and the results were given in absorbance units (ABSU). The corrected IMA formula (individual serum albumin concentration/median serum albumin concentration of the population) $\times$ IMA ABSU value was used as the final expression of IMA levels [23].

\section{Statistical analysis}

Statistical analysis was performed with the program SPSS version 11.0 for Windows (SPSS Inc., Chicago, IL). The variables were investigated using visual and analytical methods to determine whether or not they are normally distributed. Normality tests were made with the Shapiro-Wilk test. Normally distributed continuous values were expressed as mean \pm SD and categorical variables as numbers and percentages. Non-normally distributed parameters are reported as medians with interquartile range (IQR). Between-group comparisons were made with ANOVA for normally distributed variables and with the Kruskal-Wallis test for non-normally distributed variables. $P<0.05$ was accepted statistically significant.

\section{Results}

The demographic features of patient and control groups are shown in Table I and clinical features of BD patients are shown in Table II. hsCRP, TNF- $\alpha$, homocysteine, IMA, apoB/apoA1, Lp-pla2, ESR and HDL levels in patient and control groups were significantly different $(p<0.001, p=0.001$, $p<0.001, p<0.001, p<0.001, p<0.001, p=0.003$ and $p<0.001$ respectively) (Table III). In Behçet's patients with vascular involvement, homocysteine, TNF- $\alpha$ and Lp-pla2 levels were significantly higher than in Behçet's patients without vascular involvement $(p=0.035, p=0.010$ and $p<0.001$ respectively) (Table IV). No statistically significant difference was found in levels of LDL, total cholesterol and triglyceride between patient and control groups. There was no difference in ESR levels between patients with and without vascular involvement, but ESR levels of these two groups were individually different from ESR of the control group. When these parameters were evaluated in patient and control groups due to disease activity, no significant difference was found in any of them except total cholesterol ( $p=0.026$ ) (data not shown).

Table I. Demographic features of Behçet's disease and control groups

\begin{tabular}{|lcc|}
\hline Parameter & $\begin{array}{c}\text { Behçet's disease } \\
\text { group }\end{array}$ & Control group \\
\hline $\begin{array}{l}\text { Age [years], } \\
\text { mean } \pm \text { SD }\end{array}$ & $39.2 \pm 9.2$ & $41.5 \pm 11.0$ \\
\hline $\begin{array}{l}\text { Gender, } n(\%): \\
\text { Male }\end{array}$ & $33(66)$ & $18(60)$ \\
\hline Female & $17(34)$ & $12(40)$ \\
\hline
\end{tabular}




\section{Discussion}

Neutrophil hyperfunction, vasculitis and autoimmune response are the three major pathophysi-

Table II. Clinical features of patients with Behçet's disease

\begin{tabular}{|c|c|}
\hline Parameter & Values \\
\hline $\begin{array}{l}\text { Age at diagnosis [years], } \\
\text { mean } \pm \mathrm{SD}\end{array}$ & $31.5 \pm 4.6$ \\
\hline $\begin{array}{l}\text { Disease duration [months], } \\
\text { median (IQR) }\end{array}$ & $95.8(72.3)$ \\
\hline Active disease, $n(\%)$ & $27(54)$ \\
\hline BDCAF, median (IQR) & $2.0(0.0-3.0)$ \\
\hline \multicolumn{2}{|c|}{$\begin{array}{l}\text { Disease manifestations ever presented by Behçet's } \\
\text { disease patients, } n(\%) \text { : }\end{array}$} \\
\hline Oral ulcers & $50(100)$ \\
\hline Genital ulcers & $39(78)$ \\
\hline Erythema nodosum & $24(48)$ \\
\hline Papulopustular lesions & $31(62)$ \\
\hline Positivity of pathergy test & $11(22)$ \\
\hline Arthritis & $21(42)$ \\
\hline Uveitis & $20(40)$ \\
\hline Vascular involvement: & $22(44)$ \\
\hline Venous thrombosis & $21(95.5)$ \\
\hline Arterial aneurism & $1(4.5)$ \\
\hline $\begin{array}{l}\text { Gastrointestinal system } \\
\text { involvement }\end{array}$ & $1(2)$ \\
\hline Pulmonary aneurism & $1(2)$ \\
\hline
\end{tabular}

ological changes that play an important role in BD pathogenesis [2]. Vascular and cardiac complications in $\mathrm{BD}$ are brought by atherosclerosis. However, there is a limited number of studies evaluating atherosclerosis and its relationship with $\mathrm{BD}$, which remains in dispute [24, 25]. Besides being an aspect of chronic inflammatory diseases, oxidative stress is also defined to be an independent factor related to vascular wall dysfunction, even in patients who do not have any clinical signs of atherosclerosis [26]. In BD patients, most of the atherogenic, inflammatory and oxidative stress markers have been evaluated in different studies which basically compared levels of these markers in active and inactive patients [27-30]. In the current study, we evaluated lipids, lipoproteins, and various inflammatory and atherogenic parameters in BD patients with and without vascular involvement, as most of the atherogenic process takes place in the vessels, to give a general perspective of laboratory parameters in BD patients, especially to highlight vascular involvement.

Lower HDL levels in BD patients than healthy controls were observed. No significant difference was found between levels of LDL, triglyceride and total cholesterol in patient and control groups. No difference between blood lipid levels of healthy individuals was found in previous studies but Orem et al. found lower levels of blood lipids in active $\mathrm{BD}$ patients, suggesting a protector mechanism against atherosclerosis [29]. In our study, lower HDL levels in Behçet's patients may be accepted as a predictor of vulnerability to atherosclerosis.

An increase in apoB/apoA1 ratio has been shown to be associated with cardiovascular disease progression. An increased ratio of apoB/

Table III. Serum levels of laboratory parameters among patient and control groups

\begin{tabular}{|lccc|}
\hline Parameter & $\begin{array}{c}\text { Patients with } \\
\text { Behçet's disease }\end{array}$ & Healthy controls & $P$-value \\
\hline IMA (ABSU) & $0.53 \pm 0.073$ & $0.43 \pm 0.077$ & $<0.001$ \\
\hline hsCRP $[\mathrm{mg} / \mathrm{l}]$ & $6.54(9.83)$ & $0.90(1.36)$ & $<0.001$ \\
\hline TNF- $\alpha[\mathrm{pg} / \mathrm{ml}]$ & $8.67(21.62)$ & $2.56(3.52)$ & 0.001 \\
\hline Homocysteine $[\mu \mathrm{mol} / \mathrm{l}]$ & $11.82(4.61)$ & $9.63(4.13)$ & $<0.001$ \\
\hline HDL $[\mathrm{mg} / \mathrm{dl}]$ & $41.16 \pm 8.88$ & $49.5 \pm 8.6$ & 0.001 \\
\hline LDL $[\mathrm{mg} / \mathrm{dl}]$ & $105.5 \pm 33.0$ & $102.6 \pm 23.9$ & 0.15 \\
\hline Triglyceride $[\mathrm{mg} / \mathrm{dl}]$ & $119.5(54)$ & $108.0(59)$ & 0.10 \\
\hline Total cholesterol $[\mathrm{mg} / \mathrm{dl}]$ & $155.0(54)$ & $148.5(50)$ & $<0.001$ \\
\hline ApoB/apoA1 & $0.84(0.42)$ & $0.59(0.23)$ & $<0.001$ \\
\hline Lp-pla2 $[\mathrm{ng} / \mathrm{ml}]$ & $462.5(178.5)$ & $255.5(139.2)$ & 0.003 \\
\hline ESR $[\mathrm{mm} / \mathrm{h}]$ & $13.5(20.25)$ & $6.0(7.25)$ & 0.98 \\
\hline
\end{tabular}

IMA-ischemia modified albumin, hsCRP-high-sensitivity C-reactive protein, TNF- $\alpha$-tumornecrosis factor- $\alpha$, apoB/apoA1-apolipoprotein B/apolipoprotein A1, Lp-pla2 - lipoprotein-associated phospholipase A2, ESR - erythrocyte sedimentation rate. 
Table IV. Differences in serum levels of laboratory parameters according to vascular involvement

\begin{tabular}{|lcccccc|}
\hline Parameter & $\begin{array}{c}\text { Patients without } \\
\text { vascular } \\
\text { involvement } \\
(n=28)\end{array}$ & $\begin{array}{c}\text { Patients with } \\
\text { vascular } \\
\text { involvement } \\
(n=22)\end{array}$ & $\begin{array}{c}\text { Healthy controls } \\
(n=30)\end{array}$ & $P$-value* & $P$-value & $P$-value \\
\hline ESR & $16.0(9.2)$ & $30.0(8.5)$ & $12.0(2.0)$ & 0.053 & 0.096 & $<0.001$ \\
\hline IMA & $0.51 \pm 0.07$ & $0.55 \pm 0.08$ & $0.43 \pm 0.07$ & 0.160 & $<0.001$ & $<0.001$ \\
\hline hsCRP & $10.9(5.1)$ & $26.3(9.7)$ & $5.5(4.6)$ & 0.052 & $<0.001$ & $<0.001$ \\
\hline HDL & $40.8 \pm 8.5$ & $41.5 \pm 8.5$ & $49.5 \pm 8.6$ & 0.950 & 0.001 & 0.006 \\
\hline Hcy & $11.60(3.59)$ & $12.52(7.84)$ & $9.61(12.67)$ & 0.035 & $<0.001$ & $<0.001$ \\
\hline ApoB/apoA1 & $0.80(0.42)$ & $0.94(0.42)$ & $0.59(0.23)$ & 0.143 & 0.001 & $<0.001$ \\
\hline Lp-pla2 & $407(160)$ & $589(165)$ & $255(139)$ & $<0.001$ & $<0.001$ & $<0.001$ \\
\hline TNF- $\alpha$ & $2.9(18.8)$ & $18.1(25.3)$ & $2.5(3.6)$ & 0.001 & 0.110 & $<0.001$ \\
\hline
\end{tabular}

ESR - erythrocyte sedimentation rate, IMA - ischemia modified albumin, hsCRP - high-sensitivity C-reactive protein, apoB/apoA1 apolipoprotein B/apolipoprotein A1, Lp-pla2 - lipoprotein-associated phospholipase A2, TNF- $\alpha$ - tumor necrosis factor- $\alpha$. ${ }^{*} D i f f e r e n c e$ between patients with and without vascular involvement, ${ }^{* *}$ difference between patients without vascular involvement and healthy controls, ${ }^{* * *}$ difference between patients with vascular involvement and healthy controls.

apoA1 has been found in previous studies in chronic illnesses such as diabetes, coronary artery disease and some chronic inflammatory diseases including RA $[10,11,30]$. There are no previous studies in the literature evaluating apoB/apoA1 ratio in Behçet's patients. Our study is the first one to emphasize the increased ratio of apoB/ apoA1 in Behçet's patients. No differences were found between patients with and without vascular involvement. This finding is also consistent with our finding of decreased HDL levels in the patient group as it supports the tendency for atherogenicity.

Erythrocyte sedimentation rate is a conventional and non-specific marker of inflammation. So our findings of higher ESR levels in BD patients than in controls is an expected outcome. When we compared ESR values according to vascular involvement, we only found a statistically significant difference between BD patients with vascular involvement and the control group, which may point to a higher level of inflammation in the vascular group.

The TNF- $\alpha$ is one of the most well-known proinflammatory cytokines and it has been extensively studied in a wide range of inflammatory diseases. TNF- $\alpha$ 's effects on lipid metabolism may cause generation of nitric oxide to increase circulating levels of non-esterified fatty acids. TNF- $\alpha$ is known to be one of the major cytokines to play role in $\mathrm{BD}$ pathogenesis. Its effect on patients is well known and TNF- $\alpha$ blockers are used in treatment of BD patients with vascular involvement [5-7, 31]. The observation of higher TNF- $\alpha$ levels in BD patients than in the control group was an expected outcome. Additionally, TNF- $\alpha$ levels were higher in BD patients with vascular involvement. None of our pa- tients were receiving anti-TNF- $\alpha$ treatment, so our results are not affected by medication usage. Our results also suggest that TNF- $\alpha$ can be a laboratory marker of vascular involvement in BD patients.

Being a well-known acute phase reactant, CRP actively regulates the innate immune system. It is also considered to be a serum biomarker for chronic inflammation. CRP activates the classical complement pathway and inactivates the alternative pathway which is accepted as a risk factor for atherosclerosis. In recent studies, CRP and especially hsCRP were suggested as strong predictors of inverse cardiovascular events leading to myocardial infarction, being a major marker and mediator for atherosclerosis. Therefore it is expected that hsCRP levels are exacerbated in Behçet's patients compared to controls [32-34].

As oxidative stress and atherogenic dyslipidemia have a synergistic impact on atherosclerosis and CVD, Lp-pla2, being an enzyme involved in degradation of platelet activating factor (PAF), is accepted as an independent risk factor for CVD. In addition to hsCRP, Lp-pla2 has a significant role in indicating vascular inflammation and atherosclerotic plaque formation. Orem et al. investigated Lp-pla2 levels in BD patients and found that it is significantly higher in active BD patients $[9,29]$. In our study we also found higher Lp-pla2 levels in BD patients than in the control group. Also in the vascular BD group, Lp-pla2 levels were significantly higher than in patients without vascular involvement (Table IV). No previous studies in BD patients have emphasized this difference in the vascular group. Our study can be considered as a preliminary study that suggests evaluation of Lp-pla2 levels in BD patients with vascular involvement. Generally accepted 
as an oxidative stress biomarker, IMA has been extensively studied recently [24]. Elevated serum levels of IMA in patients with BD were also demonstrated in previous studies. Çapkın et al. reported significantly higher IMA levels in BD patients with vascular complications compared to patients without [28]. In our study, we found elevated IMA levels in BD patients compared to the control group. However, we did not find any significant difference between IMA levels of BD patients with and without vascular involvement.

Hyperhomocysteinemia ( $\mathrm{HHCY}$ ) is thought to lead to atherosclerosis by causing peroxidation of lipids, impairing vasomotor regulation, and causing vascular endothelial injury. $\mathrm{HHCY}$ is assumed to be an independent risk factor for coronary arterial disease, cerebrovascular disease and venous and arterial thrombosis. It has also been suggested as a risk factor for venous thrombosis in $B D$ patients. Several studies have investigated HHCY in BD patients; they all found higher levels of homocysteine in $\mathrm{BD}$ patients. Ozkan et al. reported that HHCY is seen in active BD patients. Our data also agrees with these previous findings $[16,35]$. Feki et al. found no relationship between vascular involvement and HHCY in BD patients [36]. In contrast, in our study in the vascular BD group, we found a statistically significant difference in homocysteine levels compared to patients without vascular involvement. This might suggest homocysteine as a marker of vascular involvement. When we compared the patients in terms of disease activity, we did not find any significant difference between active and inactive patients in any of these parameters (data not shown).

The major goal of the present study was to find candidate laboratory markers for discrimination between BD patients with and without vascular involvement. Our results revealed three candidate atherogenic/inflammatory markers to be used for this purpose, which are TNF- $\alpha$, Lp-pla2 and homocysteine. These findings confirm the basic hypothesis of our study of seeking laboratory parameters to be used in discrimination of BD patients with vascular involvement.

The present study has some limitations, the first being the relatively small sample size. Also the data were obtained from a single center, so it was not possible to avoid patient selection bias completely.

In conclusion, this study can be considered as a pioneer study as it cumulatively evaluates lipids, lipoproteins, and various biomarkers of inflammation, oxidative stress and atherogenicity especially in patients with vascular BD. Especially in discriminating between BD patients with and without vascular involvement, this study draws attention to the biochemical markers TNF- $\alpha$, Lp-pla2 and homocys- teine. As these three are elevated more in patients with vascular involvement, they might be used as potential laboratory markers of CVD risk evaluation in $\mathrm{BD}$ patients, along with clinical findings.

\section{Acknowledgments}

The authors appreciate the technical support given by Ayşe Bayrak Ergün and Neşe Selçuk during laboratory measurements of the parameters studied.

\section{Conflict of interest}

The authors declare no conflict of interest.

\section{References}

1. Bonfioli AA, Orefice F. Behcet's disease. Semin Ophthalmol 2005; 20: 199-206.

2. Akdeniz N, Esrefoglu M, Keleş MS, Karakuzu A, Atasoy M. Serum interleukin-2, interleukin-6, tumour necrosis factor-alpha and nitric oxide levels in patients with Behcet's disease. Ann Acad Med Singapore 2004; 33: 596-9.

3. Yurdakul S, Hamuryudan V, Yazıcı H. Behçet syndrome. Curr Opin Rheumatol 2004; 16: 38-42.

4. Mendoza-Pinto C, Garcia-Carrasco M, Jimenez-Hernan$\operatorname{dez} M$, et al. Etiopathogenesis of Behcet's disease. Autoimmun Rev 2010; 9: 241-5.

5. Sahin M, Arslan C, Naziroglu M, et al. Asymmetric dimethylarginine and nitric oxide levels as signs of endothelial dysfunction in Behcet's disease. Ann Clin Lab Sci 2006; 36: 449-54.

6. Caliskan M, Yilmaz S, Yildirim E, et al. Endothelial functions are more severely impaired during active disease period in patients with Behcet's disease. Clin Rheumatol 2007; 26: 1074-8.

7. Sari RA, Kiziltunç A, Taysi S, Akdemir S, Gündoğdu M. Levels of soluble E-selectin in patients with active Behcet's disease. Clin Rheumatol 2005; 24: 55-9.

8. Rizzo M, Kotur-Stevuljevic J, Berneis K, et al. Atherogenic dyslipidemia and oxidative stres: a new look. Transl Res 2009; 153: 217-23.

9. Örem A, Yaylı S, Arıca DA, Akcan B, Yücesan FB, Bahadır S. Lipoprotein-associate phospholipase $A 2$ level in patients with Behçet's disease. J Eur Acad Dermatol Venerol 2013; 27: 289-93.

10. Fentoglu Ö, Kırzıoglu FY, Özdem M, Kocak H, Sütcü R, Sert T. Proinflammatory cytokine levels in hyperlipidemic patients with periodontitis after periodontal treatment. Oral Dis 2012; 18: 299-306.

11. Zheng S, Han T, Xu H, et al. Associations of apolipoprotein $\mathrm{B}$ /apolipoprotein $\mathrm{A}$-I ratio with pre-diabetes and diabetes risks: a cross-sectional study in Chinese adults. BMJ Open 2017; 7: e014038.

12. Goff DC, Lloyd-Jones DM, Bennet G, et al. 2013 ACC/ AHA guideline on the assessment of cardiovascular risk. Circulation 2014; 129 (25 Suppl 2): S49-73.

13. Adukauskienė D, Čiginskienė A, Adukauskaitè A, Pentiokinienė D, Šlapikas R, Čeponienè I. Clinical relevance of high sensitivity C-reactive protein in cardiology. Medicina (Kaunas) 2016; 52: 1-10.

14. Lippi G, Montagnana M, Guidi GC. Albumin cobalt binding and ischemia modified albumin generation: an endogenous response to ischemia? Int J Cardiol 2006; 108: 410-1. 
15. Sinha MK, Roy D, Gaze DC, Collinson PO, Kaski JC. Role of "Ischemia modified albumin", a new biochemical marker of myocardial ischaemia, in the early diagnosis of acute coronary syndromes. Emerg Med J 2004; 21: 29-34.

16. Ozkan Y, Yardim-Akaydin S, Sepici A, Engin B, Sepici V, Simşek B. Assessment of homocysteine, neopterin and nitric oxide levels in Behçet's disease. Clin Chem Lab Med 2007; 45: 73-7.

17. Woo KS, Chook P, Lolin YI et al. Hyperhomocysteinemia is a risk factor for arterial endothelial dysfunction in humans. Circulation 1997; 96: 2542-4.

18. Kural-Seyahi E, Fresko I, Seyahi N, et al. The long-term mortality and morbidity of Behcet syndrome: a 2-decade outcome survey of 287 patients followed at a dedicated center. Medicine (Baltimore) 2003; 82: 60-76.

19. Fei Y, Li X, Lin S, et al. Major vascular involvement in Behçet's disease: a retrospective study of 796 patients. Clin Rheumatol 2013; 32: 845-52.

20. International Study Group for Behcet's Disease. Criteria for diagnosis of Behcet's disease. Lancet 1990; 335: 1078-80.

21. Hamuryudan V, Fresko I, Direskeneli H, et al. Evaluation of the Turkish translation of a disease activity form for Behcet's syndrome. Rheumatology (Oxford) 1999; 38: 734-6.

22. Bar-Or D, Lau E, Winkler JV. A novel assay for cobalt-albumin binding and its potential as a marker for myocardial ischemia-a preliminary report. J Emerg Med 2000; 19: 311-5.

23. Sbarouni E, Georgiadou P, Voudris V. Ischemia modified albumin changes - review and clinical implications. Clin Chem Lab Med 2011; 49; 177-84.

24. Seyahi E, Memisoglu E, Hamuryudan V, et al. Coronary atherosclerosis in Behçet's syndrome: a pilot study using electron-beam computed tomography. Rheumatology (Oxford) 2004; 43: 1448-50.

25. Hong SN, Park JC, Yoon NS, et al. Carotid artery intima-media thickness in Behçet's disease without significant cardiovascular involvement. Korean J Intern Med 2008; 23: 87-93.

26. Majer M, Gackowski D, Różalski R, Siomek-Górecka A, Oliński R, Budzyński J. Systemic oxidoreductive balance and vascular function in individuals without clinical manifestation of atherosclerosis. Arch Med Sci Atheroscler Dis 2017; 2: e37-45.

27. Bekpinar S, Kiliç N, Unlüçerçi Y, Akdag-Köse A, Azizlerli G, Ozbek-Kir Z. Evaluation of nitrosative and oxidative stress in Behçet disease. J Eur Acad Dermatol Venereol 2005; 19: 167-71.

28. Capkin E, Karkucak M, Kola M, Karaca A, Aydin Capkin A, Caner Karahan S. Ischemia-modified albumin (IMA): a novel marker of vascular involvement in Behcet's disease? Joint Bone Spine 2015; 82 68-9.

29. Orem A, Deger O, Memis O, Bahadır S, Ovalı E, Cimsit G. Lp(a) lipoprotein levels as a predictor of risk for thrombogenic events in patients with Behçet's disease. Ann Rheum Dis 1995; 54: 726-9.

30. Ozdemir R, Barutcu A, Sezgin T, et al. Vascular endothelial function and plasma homocysteine levels in Behçet's disease. Am J Cardiol 2004; 94: 522-5.

31. Kimak A, Strychaz-Dudziak M, Bachanek T, Kimak E. Lipids and lipoproteins and inflammatory markers in patients with chronic apical periodontitis. Lipids Health Dis 2015; 14: 162-70.

32. Stumpf C, Sheriff A, Zimmermann S, et al. C-reactive protein levels predict systolic heart failure and outcome in patients with first ST-elevation myocardial infarction treated with coronary angioplasty. Arch Med Sci 2017; 13: 1086-93.

33. Leitemperguer MR, Tatsch E, Kober H, De Carvalho JA, Moresco RN, Da Silva JE. Assessment of ischemia-modified albumin levels in patients with rheumatoid arthritis. Clin Lab 2014; 60: 1065-70.

34. Turhan-Caglar FM, Isiksacan N, Biyik E, Opan S, Cebe H, Akturk iF. Presepsin (sCD14-ST): could it be a novel marker for the diagnosis of ST elevation myocardial infarction? Arch Med Sci Atheroscler Dis 2017; 2: e3-8.

35. Sarican T, Ayabakan H, Turkmen S, Kalaslioglu V, Baran F, Yenice N. Homocysteine: an acitivity marker in Behçet's disease? J Dermatol Sci 2007; 45: 121-6.

36. Feki M, Houman H, Ghonnouchi M, et al. Hyperhomocysteinemia is associated with uveitis but not with deep vein thrombosis in Behçet's disease. Clin Chem Lab Med 2004; 42: 1417-23. 